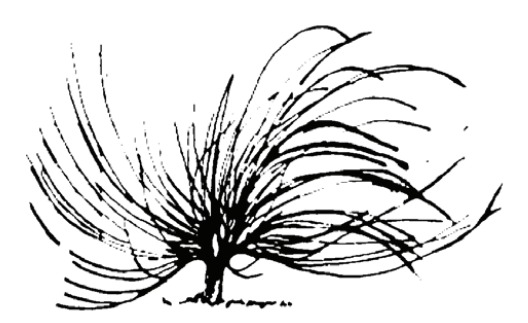

\title{
Reflexión Grupal en Torno al Concepto de Liderazgo Utilizado en Educación
}

\author{
Olga Xinia Guevara Álvarez ${ }^{1}$ \\ Universidad Nacional \\ Heredia, Costa Rica \\ olga.guevara@gmail.com
}

\begin{abstract}
Resumen
Este ensayo pretende abordar una serie de teorías en torno a la conceptualización y la puesta en práctica dentro del espacio del aula de lo que se conoce como "liderazgo educativo". Se ensaya sobre la posibilidad de generar un marco de comprensión, de reflexión, pero sobre todo de replanteamientos en relación con la participación democrática en la gestión de los procesos educativos y de aprendizaje. De esta forma y con toda antelación se deja en evidencia, desde la postura de la autora, el peligro que representa el asumir y aceptar el tradicional concepto de liderazgo, originario de la escuela empresarial (con arrogaciones de la historia fabril en relación con la servidumbre, potencialización y acumulación en la producción de capitales) y aplicarlo a la educación.
\end{abstract}

Palabras clave: educación, liderazgo, rol gestor educativo, liderazgo pedagógico, docencia

\section{Abstract}

This essay aims at addressing a number of issues related to the conceptualization and implementation of what

\section{(요 $\otimes \Theta$}

Recibido: 3 de mayo de 2015 - Aprobado: 18 de agosto de 2015

1 Docente de la División de Educología, CIDE, UNA. Coordinadora del Programa "Esperanza Joven.” Máster en pedagogía y diversidad, UNA. Licenciada en psicología, UCR. 
is known as a leadership in education. The possibility to create an understanding, reflective, and mainly, a restatement framework in relation to the democratic participation in the management of the educational processes is intended. Thus, from the author's point of view, it is considered that the traditional concept of leadership applied to education is dangerous, especially if it this concept originates from a business perspective of servitude and capital accumulation.

Keywords: education, leadership, educational manager role, pedagogical leadership, teaching

“En esta ocasión no quisiera sino averiguar cómo es posible, que tantos hombres, tantas villas, tantas ciudades, tantas naciones aguanten a veces a un tirano solo, que no tiene más poder que el que le dan, que no tiene capacidad de dañarlos, sino en tanto ellos tienen capacidad de aguantarlo, que no podría hacerles mal alguno, sino en cuanto ellos prefieren tolerarlo que contradecirlo". Etienne de La bötie

La educación superior posibilita (al menos debería posibilitar) la reflexión en torno a los procesos de aprendizaje, especialmente si el ámbito educativo universitario se construye y delimita desde el enfoque de la pedagogía crítica. Por esa razón, desde el curso conocido como "Liderazgo Académico", de la Maestría en Educación de la Universidad Nacional, se profundiza sobre el concepto, la teoría y las prácticas de aula en relación con el tema del liderazgo educativo o liderazgo académico con especial intención de revisar tanto el concepto de liderazgo educativo propuesto, como las implicaciones de este dentro del quehacer pedagógico. Sin perder de vista los posibles nuevos aportes surgidos de las construcciones de proceso durante el curso. Intención

De esta forma, doce compañeros de clase (más la docente), asumimos el reto de estudiar el concepto de liderazgo educativo y las implicaciones ideológicas que conlleva su utilización, esto como parte del curso de Liderazgo Académico que se desarrolla en la maestría. En primera instancia, nos dimos a la tarea de conocernos y de intercambiar algunas experiencias personales que definieron nuestro pensar, actuar y sentir en torno a ese concepto tan ampliamente utilizado en nuestras vidas como docentes (al menos en la mayor parte de los casos, dado que 
una estudiante del curso no era docente). Posteriormente, se plantearon algunos cuestionamientos y criterios que permitirían orientar la búsqueda de información y se procedió a realizar una amplia indagación documental. Pretendimos en ese momento crear un marco de referencia para dicha búsqueda, lo que implicó una serie de debates en clase, paralelos a las lecturas asociadas, resultando la pedagogía crítica, un punto de partida para nuestra aventura académica.

Para este momento, el grupo vive una coyuntura fundamental, están finalizando los cursos del postgrado y preparando sus proyectos finales de graduación. Este hecho es importante destacarlo especialmente porque en períodos de tanta demanda y cansancio (tal y como ellos los expresaron), fue necesario replantearse los propios espacios educativos, en función de las propias necesidades y acuerdos grupales. De esta manera, el debate se enriqueció dado que el grupo de estudiantes y la docente estuvimos dispuestos a reflexionar desde nuestro momento educativo y con el convencimiento de querer participar en la construcción y desarrollo de un curso, que en ese mismo instante nos emancipara, o que al menos nos permitiera aproximarnos a la construcción del espacio educativo, contemplando nuestras necesidades, con menos monólogos estériles, o sistemas de aprendizajes forzados, poco útiles, de respuestas superfluas y poco representativas por la premura del tiempo; entonces, se decide disfrutar del curso y permitir que la reflexión, el compromiso y la construcción grupal dieran sus frutos.

Antes de iniciar esta lectura quisiera hacer algunas observaciones que delimitan la extensión y comprensión del texto. Por un lado, se explica que no es interés del texto iniciar un análisis sobre las diferencias entre liderazgo académico y educativo, lo que resulta de fundamental interés es reconocer las posibles diferencias entre el liderazgo empresarial y educativo. Por otra parte, resulta de imperiosa importancia relatar sobre el proceso cognoscente del grupo de maestría con respecto de la reflexión del tema en cuestión.

Después de realizadas varias indagaciones sobre el tema del liderazgo educativo, surge una preocupación, la utilización del concepto de liderazgo en educación, con las mismas significaciones vinculadas al mundo mercantil y empresarial, evidenciándose incluso en algunas lecturas relacionadas con el tema educativo, esa supeditación con el clásico concepto de liderazgo. Esta situación indicó que existe una tendencia por manejar los temas educativos, sin la especificidad requerida de la 
propia disciplina. En la propuesta que hacen Raymond et al. (2005) en relación con la globalización y las implicaciones de esta en la Educación, se explica cómo adquiere un énfasis el tema de la gestión a partir de los procesos de la globalización, creándose una base legitimadora y unificadora para rediseñar nuevas burocracias estatales y se señala:

Si inicialmente existe poco debate acerca de la política educacional, es debido a que uno de los principios fundamentales de la nueva gestión es que no existe nada que distinga a la educación, esta puede ser conceptualizada y controlada como cualquier otro servicio o institución. Parte del éxito de la nueva gestión globalizada está precisamente en sus reivindicaciones de estos aspectos genéricos: su aplicabilidad a todas las esferas de la administración y homogenización de todos los problemas técnicos o institucionales como problemas de gestión. (p.81)

De esta manera, la primera preocupación lleva al grupo a otro cuestionamiento. En algunos de los textos revisados, los autores abordan el tema de liderazgo utilizando las mismas clasificaciones y análisis que realizan las empresas, pero bajo un discurso de aparente adaptabilidad a las nuevas necesidades educativas, económicas y sociales. Sin embargo, al profundizar en las lecturas (Argos y Ezquerra, 2014; Gil, Buxarrais, Muñoz, y Reyero, 2013, entre otros) se descubre en primera instancia que, el líder termina siendo un sujeto revestido de poder (otorgado este, la mayor parte de las veces, por un superior), al cual se le hacen demandas, sobre metas muy concretas que generalmente han sido definidas, por quienes han elegido o contratado a los "supuestos líderes". En segunda, se cae en cuenta de la problemática que encierra el asumir que es posible "crear" líderes, a veces incluso, procurando que el liderazgo sea una característica de la capacidad unilateral, sin consideración del otro u otros, y con la posibilidad intrínseca de determinar rumbos, guiar grupos, empresas, instituciones o cualquier tipo de agrupación humana.

En este sentido y haciendo alusión a la supuesta relación entre poder- líder, se hace fundamental retomar los criterios de Foucault, (citado por Morrow et.al., 2007), especialmente cuando el autor señala que el poder presume de libertad, de autonomía, es decir, que no es necesario exigir, forzar, atormentar para ejercer poder sobre los demás, 
para lograr que las personas actúen de forma diferente a cómo lo harían en momentos ordinarios. De esta manera, se proponen analizar algunos principios importantes propuestos por Foucault, cuando de cuestiones de poder se refiere. En relación con la gobernabilidad, entendida como al gobierno de uno mismo y de los otros, ubicando al individuo consciente, como sujeto políticamente constituido dentro de los procesos de gestión social. Foucault intentó explicar algunas preguntas: ¿quién puede gobernar?, ¿quién es gobernado? ¿qué es gobernar?, las cuales sirvieron de preámbulo para generar nuevas discusiones en clase que nos aproximaron de nuevo a considerar las intenciones subyacentes de aquellos que se consideren líderes, pues concordamos en el grupo, que el poder no es una esfera autónoma de lo político, de lo ideológico, sino más bien aspecto producto de lo actividad humana que atraviesa y se refuerza del mundo social.

Se consideran por una parte los sistemas socioeducativos, que crean, refuerzan y legitiman el concepto de liderazgo (sin importar si quiera, si es educativo o empresarial), las metas personales de quienes asumen el concepto de liderazgo educativo (contextualizándolo al trabajo de aula). Por otra parte, el cómo se asume al líder académico o educativo en función de las posibles relaciones de poder que lleva implícitas para cada miembro del grupo que estudia el concepto.

A partir de este momento, nos dedicamos a comprender algunas de las implicaciones personales, grupales, sociales, políticas, educativas, culturales que puede incidir en la comprensión de dicho término, surgiendo así la necesidad de entender por qué no deseábamos denominar a un individuo, único, exclusivo en el rol de líder o incluso de gestor educativo, tal y como se percibe en muchos de los textos consultados, tales como Medina y Gomez (2014), Alvear (2012), Uribe, (2005), entre otros.

Por el contrario, para comprender el proceso educativo, sus participantes y dinámicas debe contemplarse en todo momento, no el protagonismo o la capacidad para direccionar, sino más bien la posibilidad que tienen los participantes de organizarse, de construir, de emitir opiniones y de generar acuerdos y acciones. De forma tal que la construcción de propuestas estén vinculadas con todos los que de una u otra forma buscan las metas que los convoca, desde sus vivencias y posibilidades. Desde esta perspectiva, cobra importancia cada rol asumido, la dinámica de esos roles dentro del grupo, las habilidades asimiladas sin imponer unas sobre otras, el reconocimiento del otro como parte 
esencial del proceso, la generación de espacios de comprensión donde el enfoque de diversidad sea fundamental, pero sobre todo la asunción de que el proceso de aprendizaje se genera desde la construcción participativa, crítica y en respeto de todos los que participan.

Adelantando conclusiones, el trabajo del grupo de maestría culmina, no con una propuesta sólida e irrefutable, sino con una serie de reflexiones, construidas desde lo grupal, que inciden sobre lo personal, que enriquecen el ámbito de la educación, la transformación social, pero por sobre todo, en las prácticas personales de aula favorecen el disfrute y el aprendizaje cooperativo. Así, la reflexión grupal a nivel conceptual en consideración de las propias prácticas docentes nos permitieron repensarnos como profesionales del proceso educativo, con la posibilidad de desarrollar, construir y promover, acciones, ideas o procesos acordes a las necesidades actuales y considerando las características de los contextos.

Se hará un paréntesis en este punto para mencionar la metodología del curso de liderazgo académico de la maestría en docencia. El curso se llevó a cabo a través de doce clases presenciales, donde la participación, la reflexión, las lecturas realizadas y el debate resultaron esenciales. Como punto de partida, se consideraron las vivencias personales, como parte del autoconocimiento construido a través de los años y de las prácticas desde el quehacer docente; en segunda instancia, cada uno de los participantes hizo propuestas de lecturas, que debía compartir, leer y argumentar en cada sesión. Durante el proceso de aprendizaje, se utilizaron una serie de estrategias didácticas que incluían además de las lecturas compartidas, diarios de clase, esquemas de trabajo, videos y otras tecnologías de la información (redes sociales), estudios de casos y juegos colaborativos que fueron ampliando el marco de la discusión.

Una de las primeras experiencias de aula que se realizó fue una reflexión grupal para conocer las nociones del concepto del liderazgo de cada miembro de la clase. Lo primero que se reconoció fue que el concepto hacía una clara referencia al rol que ejerce un individuo en un determinado grupo de personas. De forma casi unánime, se concluyó en ese momento, que el concepto de líder educativo, conllevaba una serie de atribuciones que concernían más, a la consigna del "deber ser", producto de las exigencias del entorno competitivo, que a las prácticas efectivas del docente en función de la calidad del aprendizaje logrado. De igual manera, se encontró que la selección del líder, en la mayor parte de los casos, responde a la 
escogencia de un individuo revestido de poder, encubierto de una supuesta capacidad adicional, que lo diferencia del resto, que le permite además dicha "capacidad", determinar las decisiones del resto y envestido de una evidente actitud de mandato o imposición de autoridad.

Al cuestionarnos quién es un líder en la vida de cada uno de los que participábamos de la experiencia, descubrimos que cada miembro del grupo había construido una imagen que responde a esas ideas estereotipadas o preconcebidas de líderes o individuos con poder político o económico. Para citar algunos ejemplos: el presidente del país, el director de la escuela, un jugador de fútbol, el jefe empresario, la madre Teresa de Calcuta o Gandhi, entre otros citados. El término liderazgo no se vinculó con la vida cotidiana, con las historias personales, con las trayectorias comunitarias o los personajes políticos del propio país, a pesar de que las concepciones grupales del término, lo vinculan con aspectos de la cotidianidad inmediata (es decir, deben haber líderes en el trabajo, en la escuela, en la familia, entre otros). Sin embargo, si se manejaban una serie de conocimientos teóricos en función de cómo debe ser un buen líder o cómo reconocer un buen liderazgo, muy vinculado a la tendencia de definición rápida generada por algunos sitios web, como wikipedia. Al respecto señala Muijis (2011):

Hay un primer conjunto de textos, bastante numerosos, que en realidad no establecen ninguna propuesta, ni relación causal, entre el liderazgo en el aula y los resultados de aprendizaje de los alumnos. Son propuestas más nominales que normativas, es decir, se limitan a llamar liderazgo a lo que favorezca el incremento del aprendizaje de los alumnos, sin que se sepa articular las razones particulares de esa conexión. Estos textos se corresponden con “(...) lo que he llamado 'artículos de posición'. Son artículos que ni están basados en investigaciones empíricas ni en revisiones sistemáticas de la literatura, pero contienen valoraciones de posición sobre factores como el liderazgo ético o introducen nuevos conceptos de liderazgo. Muchos de estos toman la forma de “consejos para los líderes”. (p.116)

En este momento de reflexión grupal, se regresa entonces a la deliberación inicial; resultaba muy fácil hacer discurso en torno a cómo debía ser un "buen líder", incluso referido al contexto de aula. Entre 
las principales consideraciones que habíamos identificado estaban que: debía conocer lo suficiente, no sólo de manera personal sino también en el área o rol en que se desempeña, debía provocar iniciativa y mayor disposición del grupo, estimular el sentimiento de pertenencia del colectivo, fomentar el trabajo en equipo, dar un buen ejemplo al resto de los participantes, provocar en los demás acciones vinculadas al logro de las metas, entre otros criterios. Sin embargo, para este tiempo empezábamos a descubrir una serie de palabras vinculadas al concepto del liderazgo que nos dieron una luz sobre cómo estaba estructurado y asumido ese discurso en los participantes del grupo.

A continuación, se presentan algunos de criterios que surgen sobre el concepto de liderazgo y que se manejaba en el curso durante las primeras sesiones. Se observa en ellas el tipo de concepciones e incluso algunas de las contradicciones producto de lo que "debería ser un líder" y de lo que realmente resulta.

Para iniciar, uno de los compañeros se refirió al tema del autodominio, explicando que para ser un buen líder educativo resultaba fundamental este valor, en especial, porque consideraba que si no puede "dominarse" a sí mismo, jamás podrá influenciar a los otros. En este caso es preciso destacar la palabra autodominio (auto- dominio) y entonces, ¿es importante dominarse primero para dominar a otros después?, ¿el líder siempre es una persona autodominada?

Por otra parte, una compañera del grupo, hizo alusión al sentido de nobleza y de justicia que debe tener todo líder, explicando, "hace tiempo tuve un jefe, quien era un líder para mí que se aprovechaba del trabajo que yo realizaba, exponiendo en otros círculos de trabajo, mi producción, como si fuera de él". En este sentido cabe preguntarse, ¿era el jefe un líder?, ¿debe ser todo jefe un líder?, ¿por qué hablando de líderes, surge este ejemplo?

Otro de los estudiantes del grupo, planteó que un líder no puede dudar de sus decisiones, pues de esa forma estaría demostrando poca seguridad en sí mismo; lo que incidiría directamente sobre el éxito de los objetivos de aprendizaje. En esta otra apreciación, destacamos por una parte las cualidades "sobrenaturales" que debe tener el supuesto líder, pues al no tener la posibilidad de dudar, lo separa de la realidad del ser humano. Llamó la atención la palabra éxito, considerada parte esencial del discurso de las ideologías mercantiles y de producción empresarial. 
La intención de exponer las anteriores expresiones se vincula con la posibilidad de expresar la realidad de lo acontecido durante las primeras clases, desde la óptica de humildad y con la profunda pretensión de mejorar nuestros conocimientos al respecto. Cabe señalar que para la compañera docente, cada espacio de reflexión significó de igual forma, un esfuerzo sostenido por hacer aportes que facilitaran los debates y la reflexión, reconociendo en todo momento su propio proceso de cambio. De esta forma y para ir finalizando este bagaje de expresiones de aula, no porque no hubieran abundado este tipo de comentarios, sino porque consideramos que con los aportados es suficiente para exponer el panorama que se vivenciaba al inicio de este proceso, retomamos el comentario de otra de las estudiantes, quien expresó, que un aspecto clave para el desarrollo de un buen líder es justamente el tener una buena voluntad y hacer más de lo que exige o pretende de sus seguidores. En este caso, se destaca nuevamente el concepto de exigencia, que con un poco de atrevimiento, lo ligaremos al de excelencia y efectividad. Esta misma compañera hizo un exhaustivo detalle de las cualidades que debe tener un líder en relación con su personalidad, entre las principales características, debía ser, caritativo, humilde, comprensivo, leal, amable, cooperativo, buena escucha, entre otras, que en definitiva y desde cualquier perspectiva resulta un ideal difícil de lograr.

En todo momento, tanto docente como estudiantes, sugerimos lecturas que posibilitaran la ampliación del panorama, respondieran algunas de las nuevas interrogantes encontradas y generaran reflexiones sobre el tema. De esta manera y como parte de la reflexión de la sexta clase, surgen las siguientes argumentaciones: ¿qué queremos entender por liderazgo educativo? ¿Cómo se podría orientar el trabajo de re conceptualización del término, en virtud de desfavorecer el doble discurso directivo y de adiestramiento que legitima jerarquías y posiciones de poder-sumisión dentro del quehacer educativo, bajo ese concepto? ¿está el concepto de liderazgo desprovisto de intenciones manipuladoras del nuevo orden neoliberal?

Se reconoció la relación que tiene el liderazgo educativo y la intencionalidad directiva del logro de metas en un grupo. Sin embargo, el problema de esta intencionalidad no es lograr los objetivos o metas, sino los mecanismos que se dan para el alcance de ellos. Surge en este momento, el concepto de autoridad, vinculado al de obediencia y liderazgo, criterios considerados como fundamentales para el principio de 
la administración desde las jerarquías. Estos conceptos, para el grupo, forman parte de una ideología discursiva en la jerga empresarial, la cual ha sido exaltada de forma prioritaria y constante en nuestra cultura institucional y empresarial, dominada por supuesto por los valores económicos de producción y consumismo acelerado.

Es precisamente este tipo de ideología la que ha creado y malversado algunos conceptos en favor de priorizar y responder a los intereses de pocos, sobre las necesidades de muchos. Este nuevo orden, que no nos atrevemos a decir que es mundial, pero que ha fortalecido criterios de efectividad, eficiencia, inmediatez, en virtud de responder a esa producción desmedida, al consumismo inconsciente y atomizador de la cultura humana, lo que nos hizo asociar el concepto inicial de liderazgo construido en grupo, con dichas ideologías.

Para este momento, en el grupo hemos comprendido que quizás el término liderazgo, por la historia que lo define, desde su origen hasta la formas de utilización más actualizadas, no puede representar, al menos como se ha definido hasta aquí, lo que se desea lograr en el proceso de aprendizaje de aula. Entonces, el grupo se da a la tarea de profundizar más en el análisis político, socioeconómico y contextual para entender la conformación de los propios discursos sobre liderazgo.

Surge el tema de la obediencia a la autoridad jerárquica, cuyo fundamento principal está orientado bajo el principio del miedo, del temor. Miedo a perder el trabajo, a no estar en buenos términos personales y laborales con el jerarca, a posibles represalias por expresar con libertad, a no lograr ascender en los escalafones empresariales y burocráticos e incluso a perder los afectos y beneficios sociales. Sin embargo, también quedó expuesto que esta estructura de pensamiento se fundamenta en criterios sociopolíticos más profundos, que han sido asumidos históricamente y que son parte de toda una ideología global del capitalismo voraz.

Entonces, descubrimos como grupo, que todo aquello que vinculemos con las capacidades unitarias, exclusivas, individuales, de una persona, no pueden ser lo más importante del trabajo de aula. Encontramos que la literatura que representa esa forma que construimos en nuestra percepción grupal y que deseamos desarrollar en nuestras clases, está vinculada con autores que no hablan de forma específica de liderazgo, ni educativo, ni académico, ni de ningún tipo. 
La construcción y participación de todos, las dinámicas entre los roles que cada uno asume, el desplazamiento de esos roles en cada uno de los miembros sólo puede darse desde el acuerdo grupal, que busca desde la voz de los sujetos y del conocimiento, abordar el contexto áulico de manera biográfica y participativa, reflexionando acerca de las concepciones tradicionales en función de deconstruirlas, para reinventar procesos educativos, como partícipes y testigos, como comunidad aprendiente, compleja y diversa, donde debe existir la negociación, el debate y el compromiso por parte de todos. Los nuevos intereses del grupo se orientaban más hacia la pregunta de ¿cómo hablar de liderazgo y especialmente liderazgo en educación, haciendo alusión a un nuevo paradigma educativo crítico, social- participativo y ecológico, sin hacer referencia a las posturas tradicionales mercantilistas que van en detrimento de la posición ideológica del grupo?

En la octava clase, se plantearon dos posibilidades: por una parte ponerle apellido al concepto de liderazgo y llamarlo "liderazgo en educación" con el fin de hacer una clara diferencia entre el tradicional concepto y la nueva intención de vincularlo con criterios más acordes con los nuevos paradigmas educativos y las ciencias sociales, o como segunda opción plantear la utilización de otros conceptos para referirse a los procesos que deseamos definir. Eso sí se delimitó que liderazgo sería concebido como un proceso de grupo y no como un rol personal o individual. Ante esto, el líder, no se definiría únicamente por sus cualidades personales, dependería necesariamente de su participación en el grupo, del encargo y el compromiso al asumir ese rol de guía o gestionador representativo, y de los procesos de aprendizaje sobre todo del producto de estas atribuciones encomendadas por el grupo traducidas en desarrollo autónomo y consciente de la totalidad del colectivo en función de sus acuerdos y objetivos. Además, con conciencia de que la dinámica de grupo haría posible que ese rol lo puedan asumir otras personas durante el proceso de aprendizaje.

De esta manera, se asumió que el liderazgo tiene un componente transferencial que prioriza sobre el directivo o el jerárquico. Para este momento del proceso de clase, en la novena sesión, se reconoce al liderazgo educativo como un proceso participativo, de aprendizaje permanente, colaborativo, multidimensional, donde los procesos sociales (institucionales, comunales, empresariales, familiares) siempre deben ir orientados hacia el respeto de la soberanía 
de cada grupo, al derecho a la información de cada participante para la comprensión del nuevo posible concepto. Al respecto Louis (citado por Gil, F y otros 2012) señala:

Así, en la actualidad, se defienden perspectivas más aditivas, holísticas e integradoras para explicar la influencia en conjunto del liderazgo de distintos sujetos, evitando las perspectivas únicas de "agencia" por las cuales el comportamiento de los líderes se explicaría sólo en función de sus capacidades individuales, motivaciones y negociaciones. (p.3)

Reconocimos en clase los aportes de algunos autores (Maxwell, 1998; Dimmock, 2012, entre otros) en el tema del liderazgo educativo, entendido este, como la capacidad colectiva de construir para alcanzar las metas grupales, vinculado a la importancia de la representatividad de alguno de los miembros para la gestión de proceso de construcción y aprendizaje. Al respecto el modelo de liderazgo centrado en la enseñanza y el aprendizaje de Townsend y MacBeath, (2011) nos aproximó al sentir del grupo de maestría. Dejando en evidencia que no se comparte la totalidad de la propuesta.

En este sentido, el modelo de liderazgo centrado en el proceso de enseñanza y aprendizaje supera las perspectivas meramente organizativas y de gestión empresarial del liderazgo instruccional centrado en jefes y directores. Sin embargo, a pesar de que se convierte en un modelo colaborativo y compartido, no le da un papel protagónico a los estudiantes en el proceso de construcción del aprendizaje. Este modelo pondera el liderazgo enfocado en las cualidades de los docentes y directores de los centros de enseñanza en función de la efectividad y la instrucción. Al respecto Argos y Ezquerra (2014) explican que "es importante señalar que, incluso aunque potenciemos el perfil de liderazgo del profesor en el aula, tal vez, no sea el aspecto más relevante con respecto al aprendizaje de los alumnos y a su educación" (p.119).

Por otra parte, se propone un nuevo concepto para referirse a esa actitud emancipadora, propositiva, participativa y crítica del trabajo colectivo de aula, donde el gestor educativo, que bien pudiera ser el docente, logre plasmar y potenciar los intereses grupales. Asumiendo en este caso dos premisas fundamentales, por una parte, que es un concepto que le pertenece al grupo, y por ende, es dinámico, es decir, puede cambiar 
el rol gestor o de líder, durante el proceso de aprendizaje a otro individuo. Por otra parte, se asume que la gestión del proceso de enseñanza y aprendizaje es un proceso colectivo, donde cada participante construye de manera constante, comprometida y propositiva el aprendizaje, donde se destaca la necesidad de que el docente pueda generar esos espacios de participación y gestión grupal. Es decir, si hay un docente activo, que gesta procesos, que representa y orienta al grupo en sus intereses, entonces si pone de manifiesto su liderazgo en el colectivo.

A manera de conclusiones del proceso del curso de maestría en torno al concepto de líder educativo (rol gestor en educación) y liderazgo educativo, quedan las siguientes reflexiones:

Resulta fundamental generar espacios de aula, donde la creación de situaciones comunicacionales apoyadas en la significatividad, la comunicabilidad, la criticidad, el respeto sean los pilares para el debate y la reflexión. Se trata de entender los procesos de enseñanza y aprendizaje como espacios de Interacción pedagógica permanente y multilateral. Se reconoce la importancia del rol del docente en el proceso de aula, en especial, cuando el educador es capaz de gestar procesos, motivar y potencializar el aprendizaje.

Sin embargo, se concluye que para que exista dentro del aula, un verdadero proceso participativo, de construcción grupal se requiere de mucho más que de las cualidades exclusivas del docente gestor (líder), se necesita del acuerdo, la aprobación, el compromiso, la participación del resto de la colectividad. Es decir, un proceso colectivo, donde es precisamente el grupo, el que puede determinar quién o quiénes representan, encabezan, proponen o guían el proceso de aprendizaje. Se reconoce la importancia de que los docentes puedan hacer algunas de las primeras propuestas de trabajo, que puedan ser guías, facilitadores, gestores del proceso, siempre y cuando promuevan la expresión del resto, la creación o modificación de nuevas estrategias didácticas, la reflexión crítica y la transformación de la educación que responda a las necesidades sociales del contexto actual.

Por otra parte, la concepción de liderazgo apoyada en la dominancia, el adiestramiento y control por parte unilateral del docente como representante oficial del mandato ministerial, sin consideración de las necesidades o intereses de los "otros", estudiantes o comunidades, queda muy distante del rol gestor educativo (líder educativo) del que se reflexionó en el curso. Se debe aclarar que en muchas de las 
ocasiones, el logro del cumplimiento de los objetivos propuestos por un programa oficial no resulta significativo, ni útil para las realidades del colectivo de aula, por lo que puede suceder que el grupo de estudiantes decida no considerar o sentir al docente como gestor de los procesos de aprendizaje. En este caso, puede que se dé adiestramiento o instrucción, pero no aprendizaje significativo. Al respecto y como lo señala Batallán (2000), existe una lógica burocrática que contradice a la lógica pedagógica, explica que dentro de la lógica burocrática se encuentra una normativa estatutaria, que obliga al docente a cumplir con las políticas y mandatos oficiales, los cuales, en la mayor parte de los casos limita el desempeño del docente dentro de la lógica pedagógica.

Desde nuestros intereses epistemológicos y prácticos, en el aula necesitamos más propuestas democráticas y compartidas que líderes personalistas e individualistas, necesitamos más diálogo y reflexión, más relaciones horizontales y más criticidad, en pro de generar espacios de aprendizaje más significativos y consecuentes con las nuevas demandas éticas y ecológicas del planeta.

\section{Referencias bibliográficas}

Alvear, L (2012) El liderazgo institucional o liderazgo del sistema: una investigación sobre su desarrollo en Chile .EDUCAR, vol. 48, núm. 1, 2012, pp. 43-68 Barcelona, España.

Argos, J\& Ezquerra, P(2014). Liderazgo y Educación. Editorial Universidad de Cantabria. España.

Batallán, G. (2000). Lógicas contradictorias en la definición trabajo docente. El cuaderno de actuación profesional. Cuadernos de Antropología Social, número 12. (P.12) Buenos Aires.

Gil, F. Buxarrais, M. Muñoz, M y Reyero D, (2013). Liderazgo Educativo en el aula. En XXXII Seminario interuniversitario de la teoría de la Educación. Liderazgo y Educación. Recuperado de

MacBeath, J \& Townsend, T. (2012). Asesoramiento y desarrollo profesional de los docentes: procesos clave del liderazgo efectivo para la mejora de la escuela. Editor Beatriz Ávalos. Argentina. Recuperado de: http://www.uca.edu.ar/uca/common/grupo95/ files/articulos-aguerrondo-vezub-handbook-ba-espanol-asesoramiento-y-desarrollo-profesional-de-los-docentes.pdf 
Morrow, R, .Apple,M., Peters,M., Marshall,J., Fitzsimons,P., Blackmore, J., Popkewitz,T., Mc Carthy, C., Dimitriades,G., Capella, J., Stoer,S., Cortesao,L., Kellner, D.(2007).Globalización y Educación, Manual Crítico. España: Editorial Popular.

Muijis, D. (2011). Investigando el liderazgo: hacia un nuevo paradigma. Editorial International Handbook of Leadership for Learning. Londres, Springer.

Uribe, M. (2005). El liderazgo Docente en la Construcción de la Cultura Escolar de Calidad: un Desafio de Orden Superior. UNESCO ORELAC. Revista PRELAC N. 1 Año 1. P.111. 